\title{
IMPLANTAÇÃO DO GRUPO DE ATENÇÃO À SAÚDE DO IDOSO (GRASI) NO HOSPITAL DE CLÍNICAS DA UNIVERSIDADE ESTADUAL DE CAMPINAS (SP): RELATO DE EXPERIÊNCIA
}

\author{
Maria José D'Elboux Diogo* \\ Maria Filomena Ceolim* \\ Fernanda Aparecida Cintra*
}

DIOGO, M.J.D’E.; CEOLIM, M.F.; CINTRA, F.A. Implantação do grupo de atenção à saúde do idoso (grasi) no Hospital de Clínicas da Universidade Estadual de Campinas (SP): relato de experiência. Rev.latinoam.enfermagem, Ribeirão Preto, v. 8, n. 5, p. 85-90, outubro 2000.

Este trabalho visa relatar a experiência de implantação do Grupo de Atenção à Saúde do Idoso (GRASI) junto ao ambulatório de clínica médica do Hospital de Clínicas da Universidade Estadual de Campinas (SP), cuja espinha dorsal foi a elaboração e desenvolvimento de um Programa Educativo para idosos e familiares. O programa incluiu temas de interesse da clientela (memória, sono, prevenção de quedas e alterações da visão), e de interesse dos pesquisadores (autocuidado, aspectos emocionais, senescência e senilidade). Os participantes avaliaram a experiência como sendo de significativa contribuição para o seu cotidiano, bem como para aquisição de novos conhecimentos e novas amizades.

UNITERMOS: enfermagem, enfermagem geriátrica, educação em saúde, promoção da saúde, idoso

\section{INTRODUÇÃO}

O envelhecimento populacional, embora seja um fenômeno universal, apresenta características peculiares no Brasil devido à velocidade da transição demográfica em nosso país. A expectativa de vida dos brasileiros passou de 33,7 anos em 1900 para 63,5 anos em 1980, com projeções de 75,3 anos em 2025 (VERAS, 1994).

Segundo a Organização Mundial da Saúde, um país pode ser considerado estruturalmente envelhecido quando a sua taxa de idosos ultrapassa $7 \%$ da população total. Em 1980, os idosos representavam $6,3 \%$ da população brasileira, há estimativas de que, no ano 2000 , esta cifra deverá ultrapassar os $8 \%$, chegando a $13,8 \%$ em 2025 (ORGANIZACIÓN PANAMERICANA DE LA SALUD, 1993 e VERAS, 1994).

Acompanhando a transição demográfica, verifica-se a transição epidemiológica, ou seja, enquanto que anteriormente as doenças infecciosas e parasitárias eram as principais causas de morte da população, atualmente as doenças degenerativas são mais importantes neste aspecto (KINSELLA, 1994 e VERAS, 1994).

Sabemos que, apesar de constituir um processo natural, o envelhecimento não ocorre de forma homogênea. Cada idoso é um ser único que, ao longo da sua trajetória de vida, foi influenciado por eventos de natureza fisiológica, patológica, psicológica, social, cultural e econômica, os quais podem atuar sobre a qualidade de vida na velhice. Um destes fatores é a presença de afecções crônicas, que além do risco de vida, representam uma ameaça potencial à independência e à autonomia do idoso.

Os idosos tornam-se freqüentemente mais suscetíveis às doenças ou agravos à saúde, passando a ser assíduos usuários do Sistema de Saúde. Pudemos verificar este fato em trabalho realizado no Hospital de Clínicas da UNICAMP no início da década de 90, o qual demonstrou aumento do número de internações de idosos, principalmente em tratamento clínico (DIOGO \& TESTI, 1994). Devem ser estimulados estudos que contribuam para identificar estratégias visando o atendimento às demandas singulares dos idosos, incluindo o desenvolvimento de intervenções adequadas, uma vez que estes requerem abordagens específicas advindas do conhecimento dos múltiplos efeitos do envelhecimento (CAMPEDELLI, 1983).

Muitos profissionais da área de saúde,

\footnotetext{
* Enfermeira, Professor Assistente Doutor do Departamento de Enfermagem da Faculdade de Ciências Médicas da Universidade Estadual de Campinas
} 
consideram os idosos englobados na faixa etária do adulto, resultando em inexpressiva assistência desses profissionais para as particularidades da atenção ao idoso.

Existem grupos educativos dirigidos a diabéticos e hipertensos, entre outros, que parecem priorizar aspectos específicos dessas doenças, o que sugere a necessidade de serviço especializado de assistência à saúde do idoso, seja em âmbito ambulatorial ou de internação.

Há que se enfatizar ainda a atuação da enfermeira, centrada na educação para o "cuidar" da saúde, tendo como base o conhecimento das transformações associadas ao processo de envelhecimento, visando a manutenção da capacidade funcional do idoso para a realização das suas atividades diárias e o atendimento às necessidades básicas, contribuindo para que este mantenha ou alcance sua independência (CAMPEDELLI, 1983).

Em nossa instituição (Hospital das Clínicas da Universidade Estadual de Campinas), a inexistência de um atendimento especializado na assistência aos idosos, aponta para a necessidade da criação de um serviço para o desenvolvimento de atividades de acordo com as características peculiares desta faixa etária.

\section{OBJETIVO}

O objetivo deste trabalho é relatar a experiência da implantação do Grupo de Atenção à Saúde do Idoso (GRASI) junto ao ambulatório de Clínica Médica do Hospital das Clínicas da Universidade Estadual de Campinas, no município de Campinas, SP.

\section{ESTRATÉGIAS ADOTADAS}

A espinha dorsal da implantação do GRASI foi a elaboração e o desenvolvimento de um Programa Educativo voltado a idosos e seus familiares, com a finalidade de contribuir no entendimento do processo de envelhecimento, bem como de problemas ligados a este.

DELLASEGA et al. (1994) enfatizam que, no planejamento de uma atividade de ensino voltada para idosos, alguns pontos devem ser considerados, pois alterações relacionadas ao processo de envelhecimento influenciam a habilidade do idoso para aprender novos assuntos. Por exemplo, a alteração da memória para fatos recentes, bem como a presença de comprometimento sensorial (ou seja, o declínio na visão e na audição) podem dificultar a aprendizagem e a retenção de novas informações. Outros aspectos a serem considerados são os fatores sociais, econômicos e culturais, como a deficiência de sistemas de suporte à saúde, da rede social de apoio, e da escolaridade.
Vale a pena enfatizar, ainda, a importância da motivação e do interesse dos idosos e familiares para participar da proposta. Segundo DELLASEGA et al. (1994), se estes fatores não estiverem presentes, o programa educativo estará destinado ao fracasso, mesmo com todos os esforços do educador.

O reconhecimento de que os idosos não somente podem, mas também desejam receber novas informações relacionadas ao cuidado com a sua saúde, permitem à enfermeira gerontóloga assisti-los identificando as prioridades. Estas informações servem como base para a construção de um programa educativo. A capacidade do idoso para o autocuidado deve ser estimulada ao máximo, pois encoraja a sua independência e autonomia, e promove também o senso de auto-estima. No decorrer da implantação do GRASI, procuramos criar oportunidades para a troca de experiências, a identificação de dificuldades e a abordagem de aspectos emocionais dos familiares e dos idosos.

A dinâmica desse trabalho foi a seguinte:

1. triagem dos idosos atendidos no ambulatório de Clínica Médica, na especialidade Medicina Interna, durante o mês de março de 1997, quando verificamos o interesse dos mesmos em participar do grupo;

2. composição de quatro grupos, cada qual formado por cinco idosos e seus familiares;

3. implementação do Programa Educativo, que compreendeu um encontro mensal para cada grupo, às segundas-feiras, no horário de funcionamento do ambulatório (das 13:00 às 18:00 horas). Cada grupo participou de quatro encontros, com início em abril e término em julho do mesmo ano (primeiras turmas). A partir de julho foram compostos novos grupos.

A inclusão de idosos e familiares no grupo obedeceu aos critérios: idade igual ou superior a 60 anos; capacidade de comunicação verbal que permitisse a atividade em grupo; interesse em participar deste trabalho.

\section{DESENVOLVIMENTO DO PROGRAMA EDUCATIVO}

\section{1- Planejamento e implantação}

O Programa Educativo incluiu temas de interesse da clientela e outros que consideramos relevantes para o alcance dos objetivos propostos, como por exemplo, o processo de senescência e senilidade, fatores de risco e prevenção de quedas e de outros acidentes.

DELLASEGA et al. (1994) referem que o processo de enfermagem provê base para o cuidado, e pode oferecer um procedimento sistemático para o desenvolvimento de planos de ensino. Para estas autoras, 
"ensinar é mais do que dar informação, envolve todas as quatro etapas do processo de enfermagem". Assim, anteriormente ao desenvolvimento e implementação de um plano de ensino ou programa educativo, a avaliação das necessidades de aprendizagem dos idosos e seus familiares são investigadas. Os programas "pacotes", sem a avaliação diagnóstica dos clientes, resultam em prejuízo da adesão dos participantes.

Na elaboração do programa do GRASI, foram utilizadas quatro etapas que compreendem o processo ensino-aprendizagem, descritas como: avaliação inicial, planejamento, implementação e avaliação. GRASI

$1^{a}$ Reunião - Apresentação das propostas ao

Objetivos: apresentar os membros do grupo (clientela e profissionais); descrever as propostas de trabalho e as finalidades do GRASI; identificar os temas de interesse da clientela; estabelecer o cronograma de atividades; facilitar a verbalização e a discussão, entre os participantes, dos significados de "estar envelhecendo", "ser velho", e outros que permeiam o processo de envelhecimento.

Conteúdo: 1.Propostas do GRASI; 2.Definição de temas e estratégias para os futuros encontros, 3. Conceito de "ser velho".

Estratégias: 1. Apresentação individual dos participantes, quanto a nome, idade, composição familiar, residência, atividades que desempenha habitualmente e capacidade para ler e escrever; 2. Apresentação oral das propostas e finalidades do GRASI pelas autoras do trabalho; 3. Levantamento, entre os participantes, dos temas de interesse para elaboração dos conteúdos a serem desenvolvidos nas reuniões; 4. Definição do cronograma de atividades, contendo os temas a serem abordados e os horários de início e término dos encontros; 5 . Distribuição de papel sulfite em branco e lápis preto e colorido, para cada participante realizar um desenho representativo do significado de "ser velho". 6. Cada idoso verbaliza os significados que emergiram dos desenhos.

Participaram dos grupos 18 idosos, dez deles acompanhados por um familiar (com predomínio do sexo feminino), e três enfermeiras, autoras deste trabalho. Dentre os idosos, 14 eram do sexo feminino e quatro do sexo masculino. A maior parte deles pertencia à faixa etária entre 60 e 70 anos, residia com a família e eram aposentados. Houve predomínio de analfabetos, o que contribuiu para a definição das estratégias a serem utilizadas nas reuniões.

Os temas de interesse foram: Memória e envelhecimento, Alterações visuais, Prevenção de quedas, Sono e envelhecimento, A pele na velhice,
Dificuldade de movimentação e Solidão. Como o tempo disponível para o desenvolvimento do programa era curto, estabelecemos, em comum acordo com os participantes, que os quatro primeiros temas iriam compor os trabalhos do grupo.

A seguir, distribuíamos papel e lápis para a confecção do desenho representativos do que é "ser velho". Inicialmente os idosos apresentavam certa relutância para realizá-lo, justificando-a pelo analfabetismo, receio e vergonha quanto à qualidade do desenho mas, uma vez este iniciado, utilizavam vários minutos até o seu término. A maioria dos desenhos ocupava somente a metade superior das folhas de papel e eram coloridos. As figuras mais desenhadas foram: flores com ou sem vaso, e árvores ou plantas secas. Desenharam também "banco de jardim", hospital, igreja, casa, campo de futebol e cenas da vida cotidiana (trabalhos domésticos, cuidado de horta e "brincadeiras"** com os netos). Embora não fosse nosso objetivo a interpretação dos desenhos numa perspectiva psicológica, cabe ressaltar algumas explicações dadas pelos idosos.

Um dos idosos desenhou uma casa "precisando de muita reforma, principalmente no telhado", relacionando esta casa ao corpo do velho, e atribuindo à "cabeça, órgão principal do corpo", o "principal fator do esquecimento". Outro desenho que destacamos, feito por uma idosa, representava uma "velha com rosto enrugado olhando uma laranja na árvore, no ponto de ser apanhada". Sobre esta figura, a idosa comentou que "tem coisas que a gente quer fazer e as forças não dão mais, como apanhar uma fruta no pé". Referiu a presença de dificuldades para realizar tarefas domésticas. Essas colocações foram enfatizadas por outros participantes, salientando a idéia de "perda das forças". Outra idosa desenhou um hospital e verbalizou que, para ela, o envelhecimento significaria "estar no hospital, é o único lugar que (o idoso) vai passear". O enfraquecimento e a perda da beleza corporal foram comentados por meio de desenhos de pessoas com a pele enrugada e com referências quanto à fraqueza no corpo e, em conseqüência, incapacidade para realizar atividades com a mesma agilidade que possuía no passado.

Desenhado por um idoso, o campo de futebol foi explicado como representando uma das atividades de que mais gostava. Com o envelhecimento e com as alterações no seu local de moradia "tudo mudou, o campo acabou, não teve mais baile, eu fiquei velho, os outros mudaram para a cidade ou morreram e a gente fica sem nada para fazer ". O banco de jardim, outra figura desenhada, foi apontado também como um "lugar onde ficam os velhos".

** As palavras entre aspas correspondem textualmente às usadas pelos idosos 
O desenho de uma igreja e de uma bíblia foram realizados por uma senhora que referiu "A velhice é o fim, resta ler a bíblia para morrer". Associado a essa idéia de finitude, o envelhecimento também foi representado por meio de árvores ou flores secas, sobre os quais foram feitos os seguintes depoimentos: "uma árvore seca, mas com os galhos ainda, quando a gente vê uma pessoa velha, a gente vê a pessoa como uma árvore secando", e "Somos como as flores que vão envelhecendo e murchando".

Uma idosa desenhou uma casa, referindo que "Os velhos precisam de uma casa, é uma coisa mais importante da vida, ter onde morar com os filhos, não precisar de asilo. Às vezes não resolve, mesmo tendo casa os filhos jogam no asilo". A questão familiar foi apontada por vários idosos, no sentido de não se sentirem integrantes do contexto familiar, a não ser quando ajudam a cuidar dos netos.

Por outro lado alguns idosos realizaram desenhos demonstrando a presença de energia, como, por exemplo, uma pessoa "cuidando da horta", "brincando com netos ("é o maior prazer")", "cuidando do seu corpo", "tendo tempo para fazer o que gosta, como cuidar de flores" e "fazendo caminhadas". Outros comentaram ainda: "Feliz daquele que fica velhinho", "Tem muito velho enxuto".

As interpretações feitas pelos idosos revelam vários problemas decorrentes do processo de senescência, nos aspectos fisiológicos, psicológicos e sociais. A partir destas colocações, trazíamos para discussão no grupo os conceitos e preconceitos relacionados à velhice, as questões relacionadas ao início do envelhecimento, à imagem corporal e à importância do cuidado à saúde.

$2^{\mathrm{a}}$ Reunião - Tema: Memória e envelhecimento.

Objetivos: identificar as alterações da memória decorrentes do processo de envelhecimento e desenvolver estratégias para prevenir o esquecimento e facilitar a recuperação de fatos e informações.

Conteúdo: 1.Conceito de memória; 2.0 processamento da memória; 3.Causas do esquecimento; 4.Alterações com o envelhecimento; 5.Uso de medicações; 6.Medidas para prevenir o esquecimento.

Estratégias: 1. Utilização de um cartaz com recortes de revistas mostrando personalidades políticas, esportistas, artistas de décadas anteriores e do momento, objetos e bebidas mais conhecidos atualmente e divulgados pela mídia (ex. garrafa de refrigerante); 2.Uso de pequenos cartazes explicativos sobre o processamento da memória, armazenamento das informações, tipos de memória, alterações no envelhecimento, principais causas do esquecimento e estratégias para sua prevenção. 3 . Exercícios com lembranças da infância, da vida adulta e de notícias recentes veiculadas pela mídia. 4. Emprego de técnicas associativas facilitadoras da memorização dos nomes dos participantes do grupo.

O "problema do esquecimento", como foi verbalizado pelos idosos, é caracterizado como uma das alterações cognitivas mais freqüentes no envelhecimento, trazendo várias conseqüências para a realização das atividades diárias, para o relacionamento com os familiares e para o autoconceito. No grupo, os idosos relataram situações nas quais haviam vivenciado o esquecimento, bem como as experiências e estratégias utilizadas para preveni-lo.

Ao final da reunião, solicitávamos que, no próximo encontro, cada participante fizesse o relato de uma notícia ou um fato ocorrido no período entre as reuniões.

$3^{\mathrm{a}}$ Reunião - Tema: Sono e alterações no envelhecimento

Objetivos: Reconhecer as principais alterações ocorridas no sono ao longo do desenvolvimento e envelhecimento do ser humano, relacionando-as com as percebidas no seu próprio sono; identificar hábitos e fatores que contribuem para melhorar ou piorar a qualidade do sono.

Conteúdo: Características dos padrões de sono ao longo do desenvolvimento do ser humano; 2. Modificações mais freqüentes que ocorrem no sono, com o envelhecimento; 3 . Hábitos e fatores que interferem na qualidade do sono: regularidade de horários; atividade física; exposição ao sol; alimentação; ambiente apropriado para dormir; uso de fumo, álcool e bebidas com cafeína; 4.Uso de medicamentos para dormir: riscos.

Estratégias: 1.Técnicas de evocação da memória; 2. Emprego de técnica simples de relaxamento; 3. Utilização de pequenos cartazes com recortes de revistas representando hábitos e fatores que contribuem para melhorar ou piorar a qualidade do sono, que deveriam ser identificados pelos participantes; 4 . Relato de experiências em exposição oral;

No início do terceiro encontro, retomávamos o tema abordado na reunião anterior, e solicitávamos que os idosos relatassem o que fora discutido. Cada idoso apresentava também uma notícia ou fato ocorrido no período entre os dois encontros.

Quanto ao sono, a maior parte dos idosos negavam problemas crônicos relacionados. Referiam perda de sono eventual, principalmente devido a preocupações do cotidiano. O reconhecimento das figuras nos cartazes foi dificultado pela deficiência visual, para alguns participantes. Uma vez identificada a figura, relacionavam-na adequadamente com hábitos e fatores responsáveis pelo sono de melhor ou pior qualidade, e participavam ativamente relatando suas experiências. Alguns fatores não representados pelas figuras também foram apontados, como a perturbação causada no sono pelo horário de verão.

$4^{\mathrm{a}}$ Reunião - Temas: Quedas e Alterações visuais no envelhecimento 
Objetivos: identificar os fatores de risco para quedas presentes no meio ambiente e decorrentes do envelhecimento; identificar alguns recursos para a prevenção das quedas; identificar as alterações visuais presentes no envelhecimento; conhecer os cuidados necessários para a saúde ocular e a boa visão.

Conteúdo: 1. Incidência e conseqüências das quedas entre os idosos; 2. Fatores de risco para quedas; 3. Aspectos da prevenção de quedas; 4. Como se desenvolve a visão; 5.Problemas mais comuns e alterações visuais com o envelhecimento; 6.Mitos sobre a visão; 7.Higiene ocular e cuidados com os olhos.

Estratégias: 1. Relato de experiências vividas pelos idosos e acompanhantes; 2. Utilização de recortes de revistas mostrando ambientes e situações de riscos para quedas e sua prevenção; 3 . Uso de modelo didático de um globo ocular; 4. Exposição oral.

A discussão dos aspectos referentes às quedas provocou grande envolvimento dos idosos, uma vez que a maioria já havia caído em casa ou na rua. A utilização dos recortes permitiu fácil reconhecimento, por parte dos mesmos, dos fatores de risco para quedas e a associação destas com o seu ambiente domiciliar. Um dos participantes, vendo a figura de uma banheira com degraus de acesso, comentou: "Isso aqui é um perigo. Não deveria dar habite-se", demonstrando sua preocupação com os riscos que este ambiente proporciona.

Quanto às alterações visuais, os idosos manifestaram também grande interesse, particularmente à identificação, no modelo didático de globo ocular, dos problemas mais comuns e das alterações ocorridas no envelhecimento. Foi possível o esclarecimento de dúvidas relacionadas a hábitos diários, como assistir televisão a pequena distância do aparelho e com a "luz apagada", realizar atividades manuais à noite e usar colírios caseiros. Oferecemos também explicações sobre a catarata e seu tratamento, entre outras doenças oculares.

\section{2- Avaliação}

Ao final de cada reunião, fazíamos uma revisão dos aspectos principais dos temas desenvolvidos, com a finalidade de verificar a compreensão do conteúdo apresentado e a construção de novos conhecimentos. Chamávamos a atenção do grupo para as atividades a serem desenvolvidas em casa, as quais retomávamos na reunião seguinte. Solicitávamos aos idosos uma breve avaliação sobre a relevância de sua participação no encontro, sendo que a maioria deles consideravam-na importante para o enfrentamento do "dia-a-dia" e dos eventos que acompanham o processo de envelhecimento.

Na última reunião de cada grupo, realizamos uma avaliação geral, englobando todos os encontros. A maior parte dos idosos e seus acompanhantes referiram ter "apreciado a experiência" e "aprendido coisas novas", manifestando interesse em divulgar as informações para amigos e familiares. Os seguintes depoimentos revelam a importância que atribuíram ao trabalho desenvolvido pelo GRASI: "Vou imediatamente conversar com a minha filha e contar tudo", e "Nunca imaginei que alguém se importasse assim com os idosos", referindo-se às coordenadoras do grupo.

\section{CONSIDERAÇÕES FINAIS}

O programa educativo tem como premissa o envolvimento de todos os membros do grupo, por meio da integração e da interação dos profissionais com os idosos e seus familiares. A participação do familiar ou de uma pessoa próxima do idoso no programa de educação à saúde é fundamental para a compreensão das implicações que o processo de envelhecimento traz na vida diária do indivíduo, bem como de seus desdobramentos no contexto familiar.

A falta de assiduidade foi o maior problema enfrentado, sendo que houve idosos que não participaram de todos os encontros de seu grupo. Embora não conduzíssemos uma investigação formal sobre as causas deste absenteísmo, acreditamos que alguns motivos podem ser relacionados a seguir.

No modelo atual de atenção à saúde ainda são mais valorizadas as atividades curativas que visam a resolução imediata de manifestações visíveis das doenças, as quais incomodam os seus portadores. Apesar de grande parte dos idosos avaliarem como importante o trabalho proposto, a aquisição de informações sobre a prevenção de doenças ou agravos à saúde pareceu-nos não ter, para alguns deles, a mesma relevância que as relacionadas às doenças.

As dificuldades com transporte também interferiram, sobremaneira, na freqüência da participação dos idosos. Muitos vinham de outras cidades, algumas situadas a cerca de $150 \mathrm{~km}$. do município de Campinas. A dificuldade em conseguir um acompanhante foi apontada por alguns participantes como outro fator importante de absenteísmo pois, embora fossem independentes nas atividades da vida diária, muitas vezes não utilizavam meios de transporte coletivo sem uma companhia. Destacam-se ainda o esquecimento da data de comparecimento no grupo e a coincidência com consultas médicas agendadas para o mesmo dia e horário.

A despeito de todas as dificuldades, sentimo-nos estimuladas a prosseguir com o trabalho, considerando o empenho dos participantes em freqüentar o grupo e em participar das atividades propostas, traduzindo o reconhecimento de que as atividades resultavam em benefício.

A experiência levou à reformulação do GRASI, 
com o aumento do número de vagas para sete, e com a modificação do conteúdo e das estratégias de apresentação. Introduzimos, por exemplo, jogos para o tema "Memória e envelhecimento", tornando a atividade mais dinâmica e exigindo maior participação dos membros do grupo.

Considerando-se o momento de transição demográfica pelo qual o país e o mundo atravessam, o aumento rápido da população idosa e a falta de recursos destinados a esta faixa etária, acreditamos que esta proposta é uma alternativa de estratégia voltada para a educação à saúde do idoso e para o preparo dos seus familiares, visando melhor entendimento do processo de envelhecimento e das alterações que o acompanham, bem como a identificação de estratégias que contribuam no enfrentamento destas mudanças.

\section{ESTABLISHMENT OF THE GROUP FOR ELDERLY CARE (GRASI) IN A GENERAL UNIVERSITY HOSPITAL (HOSPITAL DE CLÍNICAS -UNIVERSIDADE ESTADUAL DE CAMPINAS): A REPORT OF EXPERIENCE}

This study aims at reporting the experience of establishing the Group for Elderly Care (GRASI) in an outpatient clinic at a General University Hospital (Hospital de Clinicas - Universidade Estadual de Campinas), with the goal of elaborating and developing an educative program to elderly and their families. The program approached topics requested by the clients, such as increased risk for falls, changes in memory, sleep and vision, as well as issues considered important by the researchers, such as self-care, emotional aspects, senescence and senility. The experience was evaluated as a significant contribution to elderly daily life as well as a good opportunity for acquiring new knowledge and new friendships.

\section{IMPLEMENTACIÓN DEL GRUPO DE ATENCIÓN A LA SALUD DEL ANCIANO (GRASI) EN UN HOSPITAL UNIVERSITARIO (HOSPITAL CLÍNICAS - UNIVERSIDAD ESTADUAL DE CAMPINAS): RELATO DE EXPERIENCIA}

Este estudio busca presentar la experiencia de la implantación del Grupo de Atención a la Salud del Anciano (GRASI) en el ambulatorio de Clínica Medica en Hospital Universitario (Hospital de Clínicas - Universidad Estadual de Campinas), cuya fundamentación fue la elaboración y desarrollo de un programa educativo para los ancianos y sus familiares. El programa incluyó temas de interés de la clientela (memoria, sueño, prevención de caídas y alteraciones de la visión), y de interés de los investigadores (auto-cuidado, aspectos emocionales, senectud y senilidad). En la evaluación del programa, los participantes consideraron el programa positivo para su experiencia sumado a la posibilidad de nuevos conocimientos y nuevas amistades.

TÉRMINOS CLAVES: enfermería, enfermería geriátrica, educación en salud, promoción de la salud, anciano

\section{REFERÊNCIAS BIBLIOGRÁFICAS}

01. CAMPEDELLI, M.C. Atuação de enfermagem em geriatria e gerontologia. Rev. Paul. Hosp., v. 31, n. 9/10, p. 198-200, 1983.

02. DELLASEGA, C. et al. Nursing process: teaching elderly clients. J. Gerontol. Nurs., v. 20, n. 1, p. 31-8, 1994.

03. DIOGO, M.J.D’E.; TESTI, C.V. Caracterização da população idosa internada no Hospital de Clínicas da UNICAMP no período de janeiro de 1990 à dezembro de 1992. Gerontologia, v. 2, n. 3, p. 176,1994 .
04. KINSELLA, K. Dimensiones demográficas y de salud en América Latina y el Caribe. In: ORGANIZACION PANAMERICANA DE LA SALUD. La atención de los ancianos: un desafio para los años noventa. Washington, 1994. p. 318. (Publicación científica, 546).

05. ORGANIZACIÓN PANAMERICANA DE LA SALUD Enfermeria gerontologica: conceptos para la practica. Washington, 1993. (Série PALTEX, 31).

06. VERAS, R.P. País jovem com cabelos brancos: a saúde do idoso no Brasil. Rio de Janeiro: Relume Dumará, 1994. 